leads to cancer of particular histologic types, and whether the type of HRT affects the type of cancer. Reeves et al. assessed clinical data from the Million Women Study in the UK in order to investigate these issues.

Of the 1,031,224 postmenopausal women included in the analysis, 14,102 women were diagnosed with breast cancer during the study period. Histologic type was known for 13,782 of these cancers. Women who were currently taking HRT were more than twice as likely to develop lobular, mixed ductal-lobular, or tubular cancers as women who had never taken such therapy (relative risks 2.25, 2.13, and 2.66, respectively). They were also more likely to develop ductal and mucinous cancers (relative risks 1.63 and 1.58, respectively), and lobular and ductal in situ disease. The only histologic type that was not linked to HRT was medullary cancer. Estrogen-progesterone therapy conferred a greater risk of cancer than estrogenonly therapy. The effect of HRT on invasive ductal, lobular, and tubular cancer decreases as $\mathrm{BMI}$ increases, and the authors conclude that differences in average BMI between populations might account for the variability of published data on the relationship between HRT and breast cancer. They recommend further study to confirm these results.

Original article Reeves GK et al. (2006) Hormonal therapy for menopause and breast-cancer risk by histological type: a cohort study and meta-analysis. Lancet Oncol 7: 910-918

\section{Novel emergency contraceptive proves to be effective}

Emergency contraceptives can be used to reduce the rates of abortion and unwanted pregnancy. Creinin and colleagues performed a randomized, double-blind trial to compare the efficacy and safety of two emergency contraceptive drugs: the progesterone analog levonorgestrel and a new progesterone-receptor modulator, CDB-2914.

Healthy women aged 18 years or older who had requested emergency contraception within $72 \mathrm{~h}$ of unprotected intercourse were randomly assigned to receive either a $50 \mathrm{mg}$ capsule of CDB-2914 plus a placebo capsule, or two $0.75 \mathrm{mg}$ tablets of levonorgestrel, taken $12 \mathrm{~h}$ apart. The population who were evaluable for efficacy comprised 775 women treated with CDB-2914 and 774 treated with levonorgestrel.
The post-treatment pregnancy rate (verified by high-sensitivity tests for $\beta$-human chorionic gonadotropin) was $0.9 \%$ in the CDB-2914 group (95\% $\mathrm{Cl} 0.2-1.6 \%)$ and $1.7 \%$ in the levonorgestrel group (95\% Cl 0.8-2.6\%). The treatments prevented $85 \%$ and $69 \%$ of expected pregnancies, respectively. The distribution of adverse effects was generally similar in the two groups, but nausea was reported by more CDB-2914 users than levonorgestrel users (29\% versus $24 \%, P=0.03$ ). Cycle length after treatment changed significantly in both groups.

The authors conclude that CDB-2914 is at least as effective as levonorgestrel in emergency contraception, and has the advantage of being effective in a single dose. Their suggestion that CDB-2914 might be more effective than levonorgestrel when used more than $48 \mathrm{~h}$ after intercourse is yet to be confirmed.

Original article Creinin MD et al. (2006) Progesterone receptor modulator for emergency contraception: a randomized controlled trial. Obstet Gynecol 108: 1089-1097

\section{Differential diagnosis of macroprolactinomas and nonfunctioning pituitary adenomas}

Elevated serum prolactin levels can be a symptom of either prolactin-secreting tumors (macroprolactinomas) or nonfunctioning pituitary macroadenomas that disrupt the dopaminergic inhibition of pituitary lactotroph cells. It has been suggested that serum prolactin levels $>6,000-8,000 \mathrm{mU} / \mathrm{l}$ indicate macroprolactinomas, while levels $<2,000-3,000 \mathrm{mU} / \mathrm{l}$ indicate nonfunctioning pituitary tumors. Values between 2,500 and $8,000 \mathrm{mU} / \mathrm{l}$ are considered to lie in a 'grey zone' of diagnostic uncertainty. Karavitaki and colleagues assessed whether these prolactin-based criteria for differential diagnosis-which is essential for selection of appropriate therapy - need to be redefined, and if the existence of a grey zone can be verified.

Serum prolactin levels were analyzed retrospectively in 226 patients with histologically confirmed, nonfunctioning pituitary macroadenomas. Hyperprolactinemia was observed in 87 of these 226 patients. Only three patients had serum prolactin levels above $2,000 \mathrm{mU} / \mathrm{l}$, and two of these were taking medications that could potentially increase prolactin levels.

The authors conclude that a cut-off limit of $2,000 \mathrm{mU} / \mathrm{l}$ leaves practically no patients 